\title{
Glycaemic Control in Youth and Young Adults: Challenges and Solutions
}

\section{Anuradha Khadilkar Chirantap Oza}

Hirabai Cowasji Jehangir Medical Research Institute, Jehangir Hospital, Pune, Maharashtra, 4II 00I, India
Correspondence: Anuradha Khadilkar Hirabai Cowasji Jehangir Medical Research Institute, Block V Lower Basement Jehangir Hospital, 32 Sassoon

Road, Pune, 4II00I, India

$\mathrm{Tel}+91206057004$

Email anuradhavkhadilkar@gmail.com
Abstract: Diabetes is the third most prevalent severe chronic disease of youth. Management of any chronic condition like type-1 diabetes (T1D) during adolescence, a time of rapid growth and physiological changes accompanied by important individuation and socialization processes, constitutes a major challenge for the youth, their family and the health-care team. The increasing prevalence of T1D in the adolescent age group and deteriorating glycaemic control from childhood to adolescence and youth, as well as the secular trend of worsening glycaemic control in youth and young adults with T1D, are a matter of real concern. Lack of monitoring, insufficient self-control, psychosocial factors, lack of family support and parental supervision, inadequate adherence to treatment, pubertal increase in insulin resistance and incompetent transition from paediatric to adult care are likely causes of deteriorating glycaemic control in youth. Efforts to improve insulin sensitivity by using pharmacological agents such as metformin are insufficient in resolving this problem. Interventions such as structured intervention, motivational interviews, development of youth friendly services and organization of diabetes camps for peer support can improve adherence in these individuals. Innovative technologies such as continuous subcutaneous insulin infusion and continuous glucose monitoring, comprehensive multidisciplinary teams with effective communication, parental support and supervision with planned transition from paediatric to adult care will not only reduce the risk of micro- and macrovascular complications in young adults with T1D but will also cause significant improvement in their quality of life.

Keywords: youth, adolescents, type 1 diabetes, glycemic control

\section{Introduction}

Diabetes mellitus (DM) is a chronic illness in which there are raised levels of blood glucose due to inability of the pancreas to produce insulin (type-1 diabetes) or failure to use insulin effectively (type-2 diabetes) or at times a blend of both. ${ }^{1}$ Insulin not only facilitates transport of glucose from blood to body cells where it is converted to energy but also plays a vital role in the metabolism of protein and fat. Insulin deficiency over a long duration can cause complications such as cardiovascular disease (CVD), neuropathy, nephropathy and retinopathy.

Type 1 diabetes mellitus (T1D) is caused by autoimmune destruction of $\beta$-cells of the pancreas which occurs due to a combination of genetic susceptibility and environmental triggers such as viral infections, dietary factors and toxins. ${ }^{1,2}$ The incidence of T1D is increasing globally due to non-genetic factors including environmental and lifestyle changes $^{2}$ such as rapid increase in obesity and inappropriate feeding during infancy ${ }^{3,4}$ and decrease in rate of infections. ${ }^{5}$ The overall annual incidence of T1D is estimated to be around $3 \% .{ }^{6}$ Around 1,110,100 children and adolescents of age $0-19$ years are estimated 
to be affected by T1D and approximately 128,900 are newly diagnosed every year. ${ }^{7}$ India has the highest number of children and adolescents with T1D (95.6 thousand) as well as the highest number of new cases per annum (15.9 thousand). ${ }^{7}$

The World Health Organization (WHO) classifies "young people" as those of age range 10-24 years. ${ }^{8}$ The United Nations (UN) defines youth as 15-24 years of age ${ }^{9}$ and the Society for Adolescent Health and Medicine uses the age range of 18-25 years to denote the young adult age group. ${ }^{10}$ As there is a lack of consensus definition for determining the age range to be included in the description of young adulthood, we focus our attention on a broad age group of 10-25 years. Diabetes is the third most prevalent chronic disease of youth. A study from British Columbia estimated prevalence of chronic disorders in $13-19$-year-olds to be $7 \%$ in boys and $11 \%$ in girls of which $0.07 \%$ were due to T1D, amounting to approximately $1 \%$ of the cases. ${ }^{11}$ Management of a chronic illness such as T1D during adolescence, poses a major challenge for the individual as well as the family and health-care team as it is a period of rapid growth and physiological changes along with complex individuation and socialization processes.

As glycaemic control is typically worse in adolescence, it is a critical phase to improve control and reduce microvascular complications associated with T1D. ${ }^{12}$ It has been reported by the Diabetes Control and Complications Trial and the Epidemiology of Diabetes Interventions and Complications (DCCT/ EDIC) study that an improvement in glycaemic control in children and adolescents with T1D causes reduced risk of diabetes related complications. ${ }^{13}$ Current American Diabetes Association (ADA) guidelines recommend target $\mathrm{HbA} 1 \mathrm{c}$ of $<7.5 \%$ for children and $<7.0 \%$ for young adults to maintain near normal glycaemic control while avoiding severe hypoglycaemia. ${ }^{14}$ People with T1D require appropriate daily insulin treatment, regular blood glucose monitoring, physical activity, a healthy diet, education and support for them to delay or prevent complications associated with diabetes. This is particularly difficult in early childhood as well as in adolescence. Living with T1D is a challenge for the whole family, even in countries with accessible expert medical care.

The increase in prevalence of T1D in youth along with the need for high-quality disease management, leads to an increase in cost of diabetes and may have an overwhelming effect on health-care expenditure. ${ }^{15}$ A study on HbA1c changes over time among adolescent and young adult participants in the T1D exchange clinic registry showed that the average HbAlc trajectory in the 8-18 year cohort started from $8.2 \%$ at age 8 , remained stable until age 10 years, then increased until 16-17 years, followed by another stable period. ${ }^{16}$ In this narrative review article, we focus our attention on major challenges faced by youth and young adults in maintaining glycaemic control and available solutions to promote improved glycaemic control thereby improving quality of life of youth with T1D. The major databases that we searched included PubMed, MEDLINE, Embase, and Cochrane and the search terms we used were type 1 diabetes, adolescents, youth and glycaemic control. We reviewed randomized control trials, narrative reviews and meta-analysis, recommendations, and guidelines, pertaining to glycemic control in adolescents and youth with type-1 diabetes.

\section{Type I-Diabetes and Glycaemic Control in Youth - Problem Statement}

The incidence of T1D in adolescents is on the rise. Figure 1 shows the global trend of incidence and prevalence of T1D from year 2000 to 2020 . A study noted an overall increase of $21.1 \%$ (95\% CI 15.6-27.0) in prevalence of T1D in youth. ${ }^{17}$ This increase in occurrence of T1D presents a significant burden to the health of youth as well as major clinical and public health challenges due to difficulty in diagnosis and management. ${ }^{18}$ The prevalence of T1D is tightly related with increasing age. Available data indicates a range of 1 case per 1430 children at the age of 5 years to 1 in 360 children at 16 years. $^{19}$

It has been shown by various studies that glycaemic control deteriorates during adolescence with higher HbAlc concentrations than at any other time period in the lifespan of an individual with T1D. In the DCCT study, adolescents required higher doses of insulin and still had $\mathrm{HbA1c}$ values of over $1 \%$ more than that of adults being treated with similar therapeutic approaches. ${ }^{20}$ The ADA target values of HbA1c in relation to age are illustrated in Table $1 .^{21}$

The SEARCH study classified individuals who met the ADA target as having "good" control, those with HbA1c $\geq 9.5 \%$ as "poor" control, and those with HbAlc values between the cut-offs of "good" and "poor" control as "intermediate" control. It was estimated that around $17 \%$ of youth and $29 \%$ of participants aged $19+$ with T1D had poor glycaemic control. $^{22}$ The US EXCHANGE clinic registry for T1D also suggested that only $21 \%$ of adolescents with T1D meet ADA guidelines for target HbA1c. ${ }^{12}$ These results from 


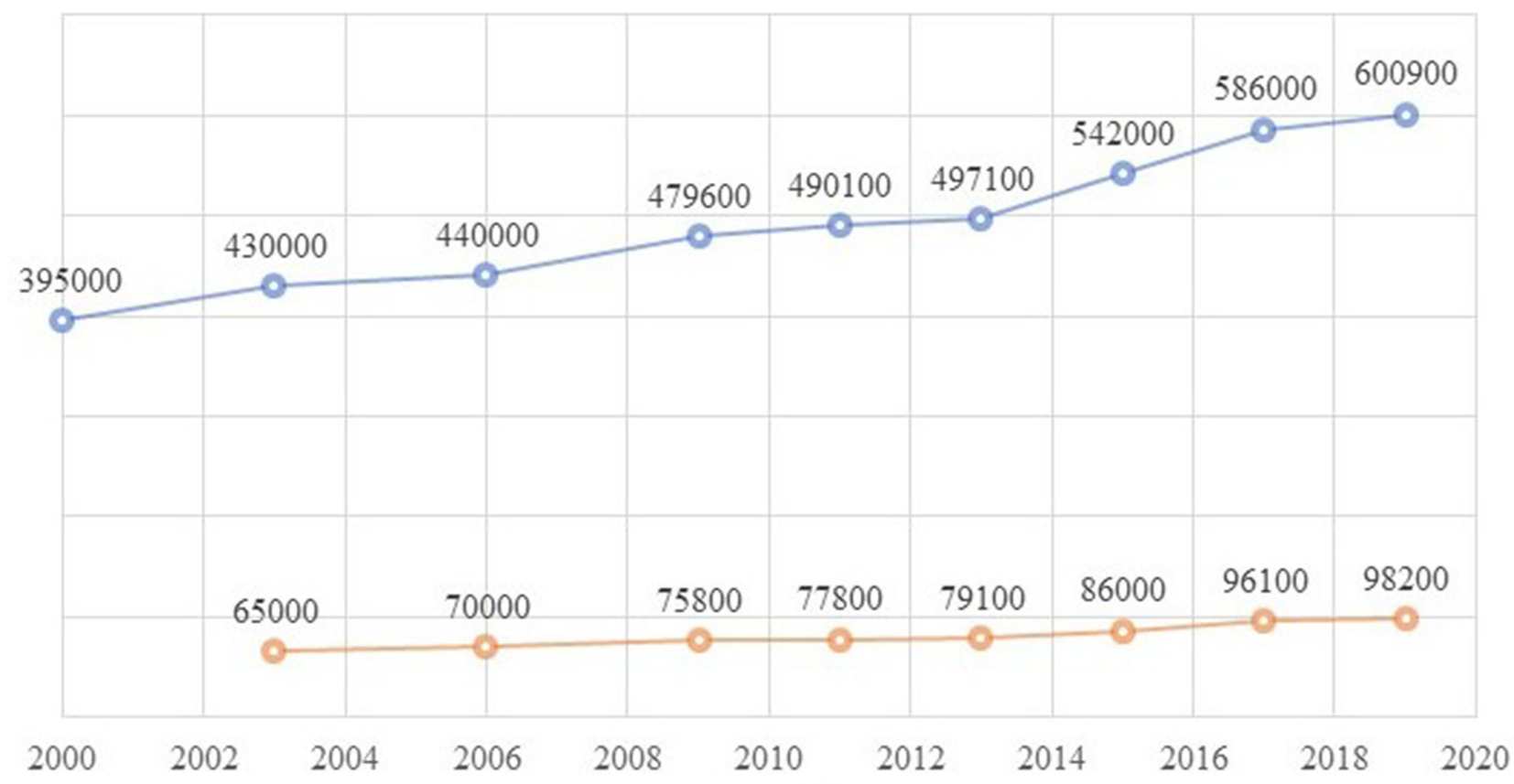

Figure I Global trend of incidence and prevalence of TID in 0-14 year age group as per IDF atlas (edition I to 9).

Europe and the USA suggest that the situation may be worse under conditions where there is limited access to insulin, selfcare tools and diabetes education, as in the low- and middleincome countries (LMIC).

A study from Saudi Arabia reports that $28.6 \%$ of toddlers achieved HbAlc of $8.5 \%, 15.6 \%$ of children achieved HbAlc of $8 \%$ and only $12.4 \%$ of adolescents achieved good glycaemic control (HbAlc 7.5\%). ${ }^{21}$ Another study from a resource-limited setup in Tanzania also noted that children in the younger age groups had significantly lower HbA1c than older children (9.8 \pm 2.0 vs $11.4 \pm 2.1) .{ }^{23}$ Although no data from India are available to compare the glycaemic control in children and adolescents with T1D on basis of their age, it is suggested that the incidence of T1D usually peaks between 10-14 years age, referred to as pubertal peaking in the Asian Indian population. ${ }^{24}$

Table I Target Values for HbAlc in Relation to Age as Described by the American Diabetes Association

\begin{tabular}{|l|l|}
\hline Target HbAlc & Age Group \\
\hline $7.5 \%$ to $8.5 \%$ & $<6$ years \\
\hline$<8.0 \%$ & 6 to 12 years \\
\hline$<7.5 \%$ & 13 to 18 years \\
\hline$<7.0 \%$ & $>19$ years \\
\hline
\end{tabular}

Abbreviation: HbAlc, haemoglobin Alc.
Moreover, the SEARCH study noted that amongst youth with T1D, mean HbA1c was $8.6 \%$ in the period 2002-2007, and statistically significantly higher in 20142019 , at $8.7 \%$, after adjustment for study site, age, sex, race/ethnicity, health insurance status, and disease duration. It was also found that mean $\mathrm{HbAlc}$ concentrations in T1D participants in the 10-14 years, 15-19 years, and 2024 years age brackets were significantly higher in the current (2014-2019) cohort than the earlier (2002-2007) cohort. $^{25}$ The proportion of youth above the target of $\mathrm{HbA1c}$ was greater with rising age during the SEARCH examination.

Thus, the increasing prevalence of T1D in the adolescent age group and deteriorating glycaemic control from childhood to adolescence and youth, as well as the secular trend of worsening glycaemic control in youth and young adults with T1D is a matter of real concern.

\section{Challenges}

Significant decline in the quality of diabetes management, treatment adherence, and glycaemic control have been observed as children reach adolescence. ${ }^{26}$ Significant gaps exist even today in understanding the pathophysiology of T1D in youth, including estimating burden and recognizing risk factors for diabetes-related complications affecting the quality of life of youth with T1D. The challenges in glycaemic control in youth and young adults are 
broadly classified as due to biological changes, inadequate compliance, psychosocial changes associated with puberty and young adulthood and transition to adult care.

\section{Biological}

Correlates of relatively high $\mathrm{HbAlc}$ include older age and longer duration of diabetes, amongst others. The deteriorating glycaemic control with increasing duration of T1D is likely due to progressive loss of beta cell function. ${ }^{27}$ Further, the hormonal changes of adolescence may lead to insulin resistance (IR). The pubertal peak occurs in conjunction with the pubertal growth spurt induced by sex steroids and pubertal growth hormone $(\mathrm{GH})$, both of which antagonize the action of insulin. In individuals without T1D, fasting and postprandial insulin concentrations have been reported to be maximum during mid to late puberty. Hyperinsulinaemic clamp techniques show a reduction of $30 \%$ in insulin sensitivity during midpuberty as compared with prepubertal and adult individuals. In individuals with T1D, a similar pattern of increase in IR has been observed, but the increase is greater than in participants without T1D. Puberty at all stages thus results in an increase in IR both in subjects with and without T1D. ${ }^{28}$ Hormonal changes that occur during puberty with increase in adiposity also contribute to poor control. ${ }^{29}$ Elevated concentrations of $\mathrm{GH}$ and reduced concentration of insulin-like growth factor (IGF) contribute to weight gain. GH also affects insulin action at a post receptor level, and the excessive GH secreted in puberty in individuals with T1D contributes to the changes in IR. Stress hormones such as cortisol and catecholamines also increase in adolescence which increase blood glucose. Moreover, at the time of puberty the lean body mass increases 2-fold over a period of 2-5 years, which causes increased insulin requirements and further contributes to inadequate glycaemic control.

\section{Adherence}

Adherence to therapeutic regimens is defined as

The extent to which a person's behaviour (in terms of taking medication, following diets, or executing lifestyle changes) coincides with medical or health advice.

Poor compliance is the leading cause of treatment failure in adolescents with T1D. ${ }^{30}$ The factors related to lack of adherence in an adolescent include cognition, perception of ailment, psychological factors, patient education, family functioning, complexity of the therapy and interference of the treatment in the patient's lifestyle. ${ }^{31}$ A study has suggested that HbAlc decreases, effect size -0.28 with $95 \%$ confidence interval (CI) -0.24 to -0.32 , with improvement in adherence. ${ }^{32}$ Around one-third of youth do not monitor $\mathrm{HbA1c}$ at recommended intervals as suggested by ADA guidelines for screening for diabetes control and complications. ${ }^{33}$ A study reports that youth with T1D rarely use continuous glucose monitoring (CGM) technology which is useful for improved glycaemic control and reduction of hypoglycaemia when used regularly. ${ }^{34}$ Another study showed that approximately $65 \%$ of patients using an insulin pump miss $>1$ bolus doses of insulin per week. It also reported that missed insulin doses in frequency of greater than $2 /$ week caused significant increase of HbAlc by $0.5 \%{ }^{35}$

\section{Psychosocial barriers}

Psychosocial barriers include a high prevalence of mood, anxiety, and depression in adolescents with T1D. Poor self-control may be related to behavioural, emotional, and cognitive changes during adolescence which contribute to poor glycaemic control. ${ }^{36}$ Even though young adults with T1D appear to be as psychologically welladjusted as their healthy peers, there may be some amount of long-standing neuropsychological effects in adolescence due to hypoglycaemia. ${ }^{37} \mathrm{~A}$ recent study found much higher rates of psychiatric morbidity in youth with duration of illness of 10 years of T1D as compared with the community. ${ }^{38}$ Eating disorders and disordered eating behaviour are reported at higher rates in adolescents with T1D. ${ }^{39}$ Also, when working with adolescents, their wishes and desires, knowledge, capabilities and rights need be taken into account. These may be constantly changing and hence require special attention which may not be provided and may add to worsening of control. In the management of T1D in younger children, management decisions are made "adult to adult" by health professionals in consultation with their parents. Day-to-day disease management is also usually performed directly by parents. Parental monitoring is a strong predictor of adolescents' adherence to management of T1D. As there is a shift in responsibility of T1D management during adolescence, a decline in parental oversight is associated with deteriorating glycaemic control. ${ }^{40}$ Also, young people and their families may give higher importance to education than treatment during late adolescence causing them to miss appointments and refusal for lunchtime treatments finally resulting in poor adherence and glycaemic control. The 
children and young adults with T1D also experience extra restriction and pain which may further contribute to undesirable outcomes. Presence of an adolescent with T1D imposes an increased burden on the parents as is the case with any chronic ailment. The demands of managing a chronic illness and the restrictions on lifestyle cause an increased dependence on family members which may be overwhelming for these young adults with T1D. ${ }^{41}$

\section{Transition from Paediatric to Adult Care}

It has been noted that odds of poor glycaemic control were 2.5 times higher among youth with T1D transitioning to adult care compared with those who remained in paediatric care. $^{42}$ Thus, challenges in the transition from paediatric to adult care in youth adds to inadequate control in youth with T1D.

\section{Solution}

There is not a "one-fits-all" solution regarding HbAlc targets and they must be individualized such that $\mathrm{HbAlc}$ is lowered without increasing frequency of episodes of nocturnal hypoglycaemia. Metformin causes improvement in vascular function and IR in adolescents with T1D. Exercise programmes tailored to decrease IR in T1D help to counteract the hostile hormonal environment affecting diabetes and resulting in complications. ${ }^{20}$

However, more than pharmacological methods to improve IR, methods to improve adherence to therapy and resolving psychosocial issues are likely to improve glycaemic control. Diabetes self-management education programmes which are structured are important for treatment of T1D; they can help to progress patients' self-management ability and prevent longterm complications. ${ }^{43}$ Structured education (SE) is thus considered as a high-quality education programme for patients with T1D. ${ }^{44}$ A review by Heller et al. recommends SE for all adolescent patients with T1D. ${ }^{45}$ A few studies also report an improvement in quality of life (QoL) in adolescents with T1D after intervention with $\mathrm{SE}$ as compared with controls. ${ }^{46,47}$ However, a meta-analysis concluded that SE had no significant effect on glycaemic control, diabetes self-efficacy, or QoL in adolescents with T1D. ${ }^{48}$ They attributed it to lack of maturity and lack of knowledge and understanding of the disease and its complications as well as the therapeutic regimens of T1D. Also, most of these SE programmes focused primarily on carbohydrate counting and insulin adjustment which may be difficult for adolescents to comprehend. Further, psychological approaches to increase motivation, compliance and confidence were not included in the SE, hence, desired results may not have been obtained.

Motivational interviewing (MI) is the technique in which the interviewer and patient engage in a concerted discussion to empower and motivate the patient to make goals for behaviour modification. ${ }^{49}$ A meta-analysis probing the effects of MI in long-lasting illnesses including T1D found that the overall effect of MI interventions on paediatric health behaviour change was small to moderate. ${ }^{50}$ Other pilot studies evaluating the effect of positive affect and cognitivebehavioural intervention with adherence to T1D therapy have shown promising results. ${ }^{51,52}$ A study also demonstrated that addition of psychoeducational intervention in managing patients with T1D had significantly improved clinic visit attendance, decreased visits to emergency department and rate of hospitalization, and an increased likelihood of improving glycaemic control and decreased risk of hypoglycaemia. ${ }^{53}$

Adherence can be improved by adjusting the doses of insulin to the patient's pubertal and growth status, adapting the treatment to the adolescent's lifestyle, and suggesting the support of siblings and peers. Keeping the same professionals in care of individual patients over time, regular assessment of adherence in a non-intimidating manner and simplified therapeutic regimen also help in improving adherence. ${ }^{54}$ As young adolescents are primarily interested in their current state and situation and are not able to envisage possible alternative futures, the treating team should provide the patient with information concerning their present condition. For example, stating that the individual might have renal defects in the future if his glycaemia is not effectively controlled may be futile. However, if it is stressed that a good glycaemic control may prevent hospitalization or improve his sports results, the adherence to therapy increases. Availability and accessibility of youth-friendly services with a safe and supportive environment are the need of the hour. Youth-friendly procedures such as time schedule not interfering with school activity, shorter waiting time, youth-friendly staff and detailed information with youth participation and community backing form crucial elements of youth-friendly services which if implemented in individuals of T1D may improve their glycaemic control. ${ }^{55}$

The USA T1D Exchange (T1DX) study group reported that around $50 \%$ of adolescents aged $13-17$ years and $52 \%$ of subjects of age 18-25 years used an insulin pump in the unit of 11,641 participants aged $<18$ years in USA. Continuous subcutaneous insulin infusion (CSII) has practical benefits over basal bolus regimen to facilitate the 
everyday management of the disease. The use of insulin pumps causes improved glycaemic control in adolescents. $^{56}$ They also improve mood, cognition and behaviour. $^{57}$ The use of continuous glucose monitoring (CGM) may be useful to avoid ideally recommended 610 fingerstick glucose checks daily by the International Society of Paediatric and Adolescent Diabetes (ISPAD). ${ }^{58}$ The application of CGM sensors for $>6$ days per week verified substantial benefit in adolescents. ${ }^{59}$ Advanced research to evaluate improvement in glycaemic control with advent of CGM with alarms, self-augmented pump therapy (SAP) and automated insulin delivery-closed loop (artificial pancreas) is being undertaken. Moreover, the previously strong association of low HbAlc with severe hypoglycaemia and coma in young individuals with T1D has decreased allowing achievement of near-normal glycaemic control in these patients. ${ }^{60}$

Diabetes camps provide many activities such as medical education talks and discussions, blood glucose testing four times a day, insulin adjustment before each meal by the team, supervision of insulin injections and planning of all meals by the dietitian. Numerous studies have revealed that diabetes camps have an advantageous effect on knowledge and self-management of T1D. ${ }^{61,62}$ Diabetes camps also improve metabolic control with potential contribution of exercise, premeditated diet, diabetes teaching, and a planned self-care environment with overseen insulin injections, blood glucose checks and peer group support for behavioural-emotional improvement. ${ }^{63}$

However, the care of adolescents with T1D surpasses the simple treatment of the disorder and should include the handling of adolescent-related health burdens and problems, precautionary intervention and counselling. A multidisciplinary team which includes various professionals such as nurses, psychologists and dietitians over and above the treating specialist may help in improving the outcome. Effective communication by health-care providers is important for adherence to therapy amongst youth with T1D. A meta-analysis showed the importance of good communication by physicians/providers on patient adherence. The relationship was even stronger when the communication was made with both parents and patients. It was also found that the odds of a patient adhering to therapy were higher (1.62) if the physician had received specific training in communication skills. ${ }^{64}$ These may be applicable for youth and young adults with T1D in improving adherence. Innovative approaches to improve glycaemic control in adolescents with T1D include the use of technology. A study that used text messages to remind youth about diabetes-related tasks demonstrated improved glycaemic control. ${ }^{65}$ A study from Israel suggests that learning with computerized simulations about biochemical processes can improve adolescents' adherence and result in improved glycaemic control. Applying scientific knowledge into the hospital educational setting may thus improve the outcome.

For better transition to adult care, the disease pathophysiology must be discussed as the young T1D patient grows up. One must recognize issues faced by the patient and their parents. Identification of contemporaries who have an interest in responsibility of young adults, a social worker who can supervise the transition, a meeting between the existing and new health-care team, follow-up phone calls and psychological backing to the patient during the transition allows smooth transition of an adolescent to adult care. ${ }^{66}$

However, one of the limitations of this review is that little information on whether an individual patient achieved his/her target is available as most studies describe the mean or median HbA1c values of the population. Moreover, studies describing variations in control between socioeconomic classes are scarce and may be a scope for future studies. To summarize, increase in prevalence of T1D, lack of monitoring, insufficient selfcontrol, psychosocial factors, lack of family support and parental supervision, inadequate adherence to treatment, pubertal increase in insulin resistance and incompetent transition from paediatric to adult care is likely to cause poor glycaemic control in youth and young adults. Proposed solutions to improve control of diabetes include deployment of a multidisciplinary team with effective communication, development of youth-friendly services, organization of diabetes camps for peer support, structured education and motivational interviews. Further, to improve adherence, promoting use of CSII, CGMS and other technological advancements may be helpful. With increasing prevalence of T1D in youth and young adults and secular deterioration of glycaemic control in this group, it has become progressively important to find ways to improve glycaemic control to prevent development of future microvascular complications and to improve quality of life. Innovative technologies, comprehensive multidisciplinary teams, parental support and supervision with planned transition from paediatric to adult care may be useful in improving glycaemic control in this vulnerable population. 


\section{Disclosure}

The authors report no conflicts of interest in this work.

\section{References}

1. Atkinson MA, Eisenbarth GS, Michels AW. Type 1 diabetes. Lancet. 2014;383(9911):69-82. doi:10.1016/S0140-6736(13)60591-7

2. Maahs DM, West NA, Lawrence JM, Mayer-Davis EJ. Epidemiology of type 1 diabetes. Endocrinol Metab Clin North Am. 2010;39 (3):481-497. doi:10.1016/j.ecl.2010.05.011

3. EURODIAB Substudy 2 Study Group. Rapid early growth is associated with increased risk of childhood type 1 diabetes in various European populations. Diabetes Care. 2002;25(10):1755-1760. doi10.2337/diacare.25.10.1755

4. Verbeeten KC, Elks CE, Daneman D, Ong KK. Association between childhood obesity and subsequent type 1 diabetes: a systematic review and meta-analysis. Diabet Med. 2011;28(1):10-18. doi:10.1111/j.1464-5491.2010.03160.x

5. Bach J-F. The effect of infections on susceptibility to autoimmune and allergic diseases. $N$ Engl $J$ Med. 2002;347(12):911-920. doi:10.1056/NEJMra020100

6. DIAMOND Project Group. Incidence and trends of childhood type 1 diabetes worldwide 1990-1999. Diabet Med. 2006;23(8):857-866. doi10.1111/j.1464-5491.2006.01925.x

7. International Diabetes Federation. IDF Diabetes Atlas. 9th ed. Brussels, Belgium; 2019. Available from: https://www.diabetesatlas. org. Accessed December 23, 2021.

8. Adolescence: A period needing special attention. Health for the world's adolescents report. Geneva: World Health Organization; 2014. Available from: http://apps.who.int/adolescent/second-decade/section2/page1/ recognizing-adolescence.html. Accessed February 19, 2016.

9. United Nations General Assembly. Convention on the Rights of the Child. Vol. 3. New York, United Nations: Treaty Ser 1989; 1577.

10. Walker-Harding LR, Christie D, Joffe A, Lau JS, Neinstein L. Young adult health and well-being: a position statement of the society for adolescent health and medicine. $J$ Adolesc Health. 2017;60 (6):758-759.

11. Suris JC, Michaud PA, Viner R. The adolescent with a chronic condition. Part I: developmental issues. Archives of disease in childhood. 2004;89(10):938-42.

12. Wood JR, Miller KM, Maahs DM, et al. Most youth with type 1 diabetes in the T1D exchange clinic registry do not meet American diabetes association or international society for pediatric and adolescent diabetes clinical guidelines. Diabetes Care. 2013;36 (7):2035-2037. doi:10.2337/dc12-1959

13. Nathan DM, Genuth S, Lachin J, et al; The Diabetes Control and Complications Trial Research Group. The effect of intensive treatment of diabetes on the development and progression of long-term complications in insulin-dependent diabetes mellitus. $N$ Engl $J$ Med. 1993;329:977-986.

14. Deiss D, Bolinder J, Riveline JP, et al. Improved glycemic control in poorly controlled patients with type 1 diabetes using real-time continuous glucose monitoring. Diabetes Care. 2006;29(12):2730-2732. doi: $10.2337 / \mathrm{dc} 06-1134$

15. Herman WH. The economic costs of diabetes: is it time for a new treatment paradigm? Diabetes Care. 2013;36:775-776. doi:10.2337/ dc13-0270

16. Clements MA, Foster NC, Maahs DM, et al. Hemoglobin A1c (HbAlc) changes over time among adolescent and young adult participants in the T1D exchange clinic registry. Pediatr Diabetes. 2016;17(5):327-336. doi:10.1111/pedi.12295

17. Dabelea D, Mayer-Davis EJ, Saydah S, et al; SEARCH for Diabetes in Youth Study. Prevalence of type 1 and type 2 diabetes among children and adolescents from 2001 to 2009. JAMA. 2014;311:1778-1786. doi:10.1001/jama.2014.3201
18. Klingensmith GJ, Pyle L, Arslanian S, et al; TODAY Study Group. The presence of GAD and IA-2 antibodies in youth with a type 2 diabetes phenotype: results from the TODAY study. Diabetes Care. 2010;33:1970. doi:10.2337/dc10-0373

19. Tuomilehto J. The emerging global epidemic of type 1 diabetes. Curr Diab Rep. 2013;13(6):795-804. doi:10.1007/s11892-013-0433-5

20. Chowdhury S. Puberty and type 1 diabetes. Indian $J$ Endocrinol Metab. 2015;19(Suppl 1):S51. doi:10.4103/2230-8210.155402

21. Al Zahrani AM, Al Shaikh A. Glycemic control in children and youth with type 1 diabetes mellitus in Saudi Arabia. Clin Med Insights Endocrinol Diabetes. 2019;12:1179551418825159. doi:10.1177/ 1179551418825159

22. Petitti DB, Klingensmith GJ, Bell RA, et al; SEARCH for Diabetes in Youth Study Group. Glycemic control in youth with diabetes: the SEARCH for diabetes in Youth Study. J Pediatr. 2009;155(5):668672.e1-e3. doi:10.1016/j.jpeds.2009.05.025

23. Noorani M, Ramaiya K, Manji K. Glycaemic control in type 1 diabetes mellitus among children and adolescents in a resource limited setting in Dar Es Salaam-Tanzania. BMC Endocr Disord. 2016;16(1):1-8. doi:10.1186/s12902-016-0113-y

24. Praveen PA, Madhu SV, Viswanathan M, et al. Demographic and clinical profile of youth onset diabetes patients in India-results from the baseline data of a clinic based registry of people with diabetes in India with young age at onset-[YDR-02]. Pediatr Diabetes. 2021;22 (1):15-21. doi:10.1111/pedi.12973

25. Malik F, Katherine A, Sauder SI, et al. Trends in glycemic control among youth with diabetes: the search for diabetes in Youth Study. Diabetes. 2020;69(Supplement 1). doi:10.2337/db20-347-OR

26. Kovacs M, Goldston D, Obrosky S, Iyengar S. Prevalence and predictors of pervasive noncompliance with medical treatment among youths with insulin-dependent diabetes mellitus. J Acad Child Adolesc Psychiatry. 1992;31:1112-1119. doi:10.1097/00004583199211000-00020

27. Scholin A, Bjorklund L, Borg $\mathrm{H}$, et al. Islet antibodies and remaining beta-cell function 8 years after diagnosis of diabetes in young adults: a prospective follow-up of the nationwide Diabetes Incidence Study in Sweden 5. J Intern Med. 2004;255:384-391. doi:10.1046/j.13652796.2003.01273.x

28. Amiel SA, Sherwin RS, Simonson DC, Lauritano AA, Tamborlane WV. Impaired insulin action in puberty. A contributing factor to poor glycemic control in adolescents with diabetes. $N$ Engl J Med. 1986;315:215-219. doi:10.1056/NEJM198607243150402

29. Goran MI, Gower BA. Longitudinal study on pubertal insulin resistance. Diabetes. 2001;50:2444. doi:10.2337/diabetes.50.11.2444

30. Scarfone R, Zorc J, Capraro G. Patient self-management of acute asthma: adherence to national guidelines a decade later. Pediatrics. 2001;108:1332-1338. doi:10.1542/peds.108.6.1332

31. Burroughs T, Pontious S, Santiago J. The relationship among six psychosocial domains, age, health care adherence, and metabolic control in adolescents with IDDM. Diabetes Educ. 1993;19:396-402. doi:10.1177/014572179301900506

32. Hood KK, Peterson CM, Rohan JM, Drotar D. Association between adherence and glycemic control in pediatric type 1 diabetes: a meta-analysis. Pediatrics. 2009;124(6):e1171-e1179. doi:10.1542/ peds.2009-0207

33. Waitzfelder B, Pihoker C, Klingensmith G, et al; SEARCH for Diabetes in Youth Study Group. Adherence to guidelines for youths with diabetes mellitus. Pediatrics. 2011;128:531-538. doi:10.1542/ peds.2010-3641

34. Wong JC, Foster NC, Maahs DM, et al. Real-time continuous glucose monitoring among participants in the T1D exchange clinic registry. Diabetes Care. 2014;37(10):2702-2709. doi:10.2337/dc14-0303

35. Burdick J, Chase HP, Slover RH, et al. Missed insulin meal boluses and elevated hemoglobin A1c levels in children receiving insulin pump therapy. Pediatrics. 2004;113(3 Pt 1):e221-e224. doi:10.1542/ peds.113.3.e221 
36. Casey BJ. Beyond simple models of self-control to circuit-based accounts of adolescent behavior. Ann Rev Psychol. 2015;66 (1):295-319. doi:10.1146/annurev-psych-010814-015156

37. Rovet J, Ehrlich R. The effect of hypoglycemic seizures on cognitive function in children with diabetes: a 7-year prospective study. J Pediatr. 1999;134(4):503-506. doi:10.1016/S0022-3476(99)70211-8

38. Northam EA, Matthews LK, Anderson PJ, Cameron FJ, Werther GA. Psychiatric morbidity and health outcome in type 1 diabetes-perspectives from a prospective longitudinal study. Diabet Med. 2005;22 (2):152-157. doi:10.1111/j.1464-5491.2004.01370.x

39. Hanlan ME, Griffith J, Patel N, Jaser SS. Eating disorders and disordered eating in type 1 diabetes: prevalence, screening, and treatment options. Curr Diab Rep. 2013;13(6):909-916. doi:10.1007/s11892-013-0418-4

40. Ingerski LM, Anderson BJ, Dolan LM, Hood KK. Blood glucose monitoring and glycemic control in adolescence: contribution of diabetes-specific responsibility and family conflict. $J$ Adolesc Health. 2010;47(2):191-197. doi:10.1016/j.jadohealth.2010.01.012

41. Eiser C, Berrenberg J. Assessing the impact of chronic disease on the relationship between parents and their adolescents. J Psychosom Res. 1995;39(2):109-114. doi:10.1016/00223999(94)00099-Q

42. Lotstein DS, Seid M, Klingensmith G, et al; SEARCH for Diabetes in Youth Study Group. Transition from pediatric to adult care for youth diagnosed with type 1 diabetes in adolescence. Pediatrics. 2013;131 (4):e1062-e1070. doi:10.1542/peds.2012-1450

43. Sherifali D, Bai J-W, Kenny M, Warren R, Ali MU. Diabetes self-management programmes in older adults: a systematic review and meta-analysis. Diabet Med. 2015;32(11):1404-1414. doi:10.1111/dme. 12780

44. Chatterjee S, Davies MJ, Heller S, Speight J, Snoek FJ, Khunti K. Diabetes structured self-management education programmes: a narrative review and current innovations. Lancet Diabetes Endocrinol. 2018;6(2):130-142. doi:10.1016/S2213-8587(17)30239-5

45. Heller SR. Structured education in type 1 diabetes. $\mathrm{Br} J$ Diabetes Vasc Dis. 2009;9(6):269-272. doi:10.1177/1474651409351646

46. Graue M, Wentzel-Larsen T, Hanestad BR, Sovik O. Evaluation of a programme of group visits and computer-assisted consultations in the treatment of adolescents with type 1 diabetes. Diabet Med. 2005;22(11):1522-1529. doi:10.1111/j.1464-5491.2005.01689.x

47. Price KJ, Knowles JA, Fox M, et al. Effectiveness of the Kids in Control of Food (KICk-OFF) structured education course for 1116 year olds with type 1 diabetes. Diabet Med. 2016;33 (2):192-203. doi:10.1111/dme.12881

48. Liu F, Guan Y, Li X, et al. Different effects of structured education on glycemic control and psychological outcomes in adolescent and adult patients with type 1 diabetes: a systematic review and meta-analysis. Int J Endocrinol. 2020;26:2020.

49. Miller WR, Rollnick S. Motivational Interviewing: Helping People Change. 3rd ed. New York: Guilford Press; 2013.

50. Gayes LA, Steele RG. A meta-analysis of motivational interviewing interventions for pediatric health behavior change. J Consult Clin Psychol. 2014;82(3):521-535. doi:10.1037/a0035917

51. Jaser SS, Patel N, Rothman RL, Choi L, Whittemore R. Check it! A randomized pilot of a positive psychology intervention to improve adherence in adolescents with type 1 diabetes. Diabetes Educ. 2014;40(5):659-667. doi:10.1177/0145721714535990
52. Salamon KS, Hains AA, Fleischman KM, Davies WH, Kichler J. Improving adherence in social situations for adolescents with type 1 diabetes mellitus (T1DM): a pilot study. Prim Care Diabetes. 2010;4 (1):47-55. doi:10.1016/j.pcd.2009.10.003

53. Svoren BM, Butler D, Levine BS, Anderson BJ, Laffel LM. Reducing acute adverse outcomes in youths with type 1 diabetes: a randomized, controlled trial. Pediatrics. 2003;112(4):914-922. doi:10.1542/peds.112.4.914

54. Anderson B, Ho J, Brackett J, et al. Parental involvement in diabetes management tasks: relationships to blood glucose monitoring adherence and metabolic control in young adolescents with insulin dependent diabetes mellitus. J Paediatr Child Health. 1997;130:257-265.

55. McIntyre P. Adolescent Friendly Health Services. Geneva: World Health Organisation; 2003.

56. Pankowska E, Blazik M, Dziechciarz P, Szypowska A, Szajewska H. Continuous subcutaneous insulin infusion vs. multiple daily injections in children with type 1 diabetes: a systematic review and meta-analysis of randomized control trials. Pediatr Diabetes. 2009;10(1):52-58. doi:10.1111/j.1399-5448.2008.00440.x

57. Knight S, Northam E, Donath S, et al. Improvements in cognition, mood and behaviour following commencement of continuous subcutaneous insulin infusion therapy in children with type 1 diabetes mellitus: a pilot study. Diabetologia. 2009;52(2):193-198. doi:10.1007/s00125-0081197-3

58. DiMeglio LA, Acerini CL, Codner E, et al. ISPAD clinical practice consensus guidelines 2018: glycemic control targets and glucose monitoring for children, adolescents, and young adults with diabetes. Pediatr Diabetes. 2018;19(Suppl 27):105-114. doi:10.1111/pedi.12737

59. Beck RW, Buckingham B, Miller K, et al. Factors predictive of use and of benefit from continuous glucose monitoring in type 1 diabetes. Diabetes Care. 2009;32(11):1947-1953.

60. Karges B, Rosenbauer J, Kapellen T, et al. Hemoglobin A1c levels and risk of severe hypoglycemia in children and young adults with type 1 diabetes from Germany and Austria: a trend analysis in a cohort of 37,539 patients between 1995 and 2012. PLoS Med. 2014;11(10):e1001742. doi:10.1371/journal.pmed.1001742

61. Mancuso M, Caruso-Nicoletti M. Summer camps and quality of life in children and adolescents with type 1 diabetes. Acta Biomed. 2003;74(Suppl. 1):35-37.

62. Semiz S, Bilgin UO, Bundak R, Bircan I. Summer camps for diabetic children: an experience in Antalya, Turkey. Acta Diabetol. 2000;37:197-200. doi:10.1007/s005920070005

63. Wang YCA, Stewart S, Tuli E, White P. Improved glycemic control in adolescents with type 1 diabetes mellitus who attend diabetes camp. Pediatr Diabetes. 2008;9:29-34. doi:10.1111/j.13995448.2007.00285.x

64. Zolnierek KB, Dimatteo MR. Physician communication and patient adherence to treatment: a metaanalysis. Med Care. 2009;47 (8):826-834. doi:10.1097/MLR.0b013e31819a5acc

65. Mulvaney SA, Anders S, Smith AK, Pittel EJ, Johnson KB. A pilot test of a tailored mobile and web-based diabetes messaging system for adolescents. $J$ Telemed Telecare. 2012;18(2):115-118. doi: $10.1258 /$ jtt.2011.111006

66. Blum R. Improving transition for adolescents with special health care needs from pediatric to adult centered health care. Pediatrics. 2002;110:1301. doi:10.1542/peds.110.S3.1301 


\section{Publish your work in this journal}

Diabetes, Metabolic Syndrome and Obesity: Targets and Therapy is an international, peer-reviewed open-access journal committed to the rapid publication of the latest laboratory and clinical findings in the fields of diabetes, metabolic syndrome and obesity research. Original research, review, case reports, hypothesis formation, expert opinion and commentaries are all considered for publication. The manuscript management system is completely online and includes a very quick and fair peer-review system, which is all easy to use. Visit http://www.dovepress.com/testimonials.php to read real quotes from published authors. 